unknown, the low abundance of carbonate fossils may reflect only the dilution of the fossil flux by ice-rafted sediment particles. It may also be that the freshwater layer accompanying Heinrich events renders the environment more hostile to calcareous plankton than to diatoms.

Despite the wealth of new information on the Heinrich layers now becoming available, there are many unanswered questions. Indications that similar sediment layers are found further north and can be traced to sources in the Fennoscandian, Barents and Svalbard ice sheets need to be put on solid footing. If Heinrich layers were deposited from other ice sheets, at the same time as the ones in the North Atlantic, this will be an important new facet of ice-age dynamics in general.

The pacing of the Heinrich layers is different from the changes in the Earth's orbit that lie behind the Milankovitch mechanism of climate change, but Bond et al. are perhaps hasty in dismissing orbital forcing as a cause of the events. The instability responsible evidently requires that ice sheets have reached a certain size, and so the layers can arise only when orbital forcing has driven the system to an unstable mode. Tracing the deposits to the earliest examples and looking for changes over the duration of the ice ages will clearly be useful. With the quick, nondestructive core-logging techniques now in use and the distinctive mineralogy of the layers, this should not be such a tall order.

Eystein Jansen is in the Department of Geology, University of Bergen, Allegaten 41, N-5007 Bergen, Norway.

\footnotetext{
1. Bond, G. et al. Nature 360. 245-249 (1992).

2. Heinrich, H. Quat. Res. 29, 142-145 (1988)

3. Ruddiman. W. Geol. Soc. Am. Bull 88, 1813-1827 (1977).

4. Lehman, S. \& Keigwin, L. D. Nature 356, 757-762 (1992).

5. Karpuz, N. K. \& Jansen, E. Paleoceanography 7, 499 520 (1992)

6. Johnsen, S. J, et al. Nature 359, 311-313 (1992).

7. Mangerud, J. et al. Quat. Res. 38, 1-31 (1992)

8. Sancetta, C. Nature 360, $249-251$ (1992).
}

\section{Waning star}

THE young stars of the Hyades cluster were found, in observation ten years ago by the Einstein satellite, to have X-ray luminosities up to 50 times higher than that of the Sun, a result presumed to indicate higher magnetic and chromospheric activity. Now the more sensitive ROSAT X-ray satellite has surveyed the cluster again, and finds some curious changes (R. A. Stern et al. Astrophys. J. 399, L159-L162; 1992). Some stars which Einstein found to be similar to one another in X-ray brightness now appear quite different, and in one case a star that was easily detected ten years ago is not seen at all. The Hyades stars are not just more active than the Sun, but apparently more variable too, going through cycles like the 11-year solar cycle and perhaps, in the one case, entering into a quiescent phase like the seventeenth-century Maunder Minimum, when no sunspots were seen for decades, and the world experienced the little ice age.

\section{One step back}

WHY does actin hydrolyse ATP when it polymerizes? What does it need the nucleotide for anyway? Two years ago a paper in Nature offered an answer. It was that the elastic properties of filaments of ADP-actin and ATP-actin differ, the former storing elastic energy for use in later processes. But now T. D. Pollard and his colleagues (J. biol Chem. 267, 20339 $20345 ; 1992$ ) assert that this attractive scheme is a figment due to the instability of ADP-actin: if the protein is treated with proper respect, they say, the differences in elasticity and viscosity of the two types of filament vanishes. Their answer to the nucleotide enigma is that conversion of ATP- to ADP-actin allows for more rapid depolymerization of the filaments when the cell no longer has need of them.

\section{First blood}

INCISOR morphology is at the cutting edge of an explanation of why vampire bats are found only in the Neotropics (M. B. Fenton Biol. J. Linn. Soc. 47, 161-171; 1992). Blood-feeding could have developed from feeding on maggots in wounds, an idea that assumes a plentiful supply of large, accident-prone mammals and birds. From this to wounding with teeth would be a small step. But why just in the Neotropics? Heritage is the key, says Fenton. The three genera of vampire all belong to the family Phyllostomidae, a diverse group confined to the New World.

Phyllostomids combine catholic tastes with large, cutting incisors, whereas most other bats have very small incisors. The capacity to make new wounds may explain the dietary shift from blowflies to blood, and the absence of vampires in Transylvania. female clones.

Polyembryonic reproduction is wide- 